\title{
The Use of NTFPs in the Baka and Kounabembe Traditional Pharmacopoeia in the Northern Periphery of the Boumba-Bek National Park (Southeastern Cameroon)
}

\author{
TAJEUKEM Vice Clotèxe ${ }^{1 *} \quad$ GUEDJE Nicole Marie ${ }^{2} \quad$ BETTI Jean Lagarde ${ }^{3}$, \\ MADOUNTSAP TAGNANG Nadège ${ }^{3} \quad$ KOYEU TATOU Emmanuel ${ }^{4}$ \\ 1.Department of Plant Science, Faculty of Science, University of Buea, Cameroon \\ 2.Department of pharmacy, Faculty of Medicine and Biomedical Sciences, University of Yaoundé1, Cameroon \\ 3.Department of Plant Biology, Faculty of Science, University of Douala, Cameroon \\ 4.HTTC, University of Bamenda, Cameroon
}

\begin{abstract}
Forest conservation around protected areas includes among others sustainable management of natural resources. This study was conducted in the area of the northern outskirts of the Boumba-Bek National Park in order to evaluate the potential use of plants in traditional medicine. Ethnobotanical surveys were conducted with local people using AFLORA survey forms. A total of 132 plant species used in traditional medicine for primary health care were identified. These plants belong to 109 genera and 45 families. 224 treatments for 101 disease symptoms were collected. These diseases include the nervous system, excretory system and nutritional function. The most recurrent diseases are cough, sexual weakness, back pain, abscess, general tiredness, malaria. Some plants such as Baillonella toxisperma, Alstonia boonei, Annonidium mannii, Ricinidendron heudelotii, Klainedoxa gabonensis, Scorodophloeus zenkeri, Swartzia sp. treat several diseases at once. The plant parts mostly used are stem bark, leaves, seeds, sap, the marrow of the bark, the pith of the stem. The main method of preparation of these recipes is decoction while the most common method of administration is oral administration. The plant resources of this village have a high potential in traditional medicine for primary health care. The importance index varied from plant to plant.
\end{abstract}

Keywords: NTFPs, AFLORA, medicinal plants, traditional medicine, primary health care.

DOI: $10.7176 / \mathrm{JBAH} / 11-4-07$

Publication date: February $28^{\text {th }} 2021$

\section{Introduction}

African forests cover a surface area of close to 241 million hectares (FAO, 2003) and the Congo Basin is the second largest tropical rain forest area in the world after Amazonia that is $12 \%$ of the tropical forest area (Tchatat $\&$ Ndoye, 2006). In addition to this dimensional importance, this forest is endowed with an exceptional biological diversity that has been preserved for ages through a rational exploitation. This has enabled the ecosystems to maintain a more or less stable balance (Koyeu et al., 2014; FAO, 2013; Nkongmeneck et al., 2007; Helms, 1998). Cameroon also belongs to this bloc and is endowed with 22.5 million hectares of rain forests that make up $48 \%$ of the national territory and which contain close to 10000 floristic species (Fongnzossie et al., 2014). Populations living in these forest areas have been exploiting these resources for ages with remarkable empirical knowhow in solving their health and nutritional problems as well as meeting their daily needs (FAO, 2015; Jamnadass et al., 2011; Sunderland, 2011).

Traditional medicine, who quality, security and efficiency are undeniable (Salmerón-Manzano et al., 2020; Orch et al., 2015; Abouri et al., 2012; Nkongmeneck, 2007), also contribute in the realisation of the universal objective of easy access to healthcare. Its efficiency stems from the fact that it is less expensive and really effective (Ouhaddou et al., 2014; WHO, 1978; 2013). Widespread knowledge within the domain of primary healthcare is still confined in the ancestral traditional cultures and is yet to be revealed because of limited research and the poor appreciation of this form of medicine. These rich biodiversities are being studied only by the scientific systems of the Western world, where researchers always extract molecules from them in order to produce drugs (Nicolas et al. 2004).

The context of globalization, coupled with the development of ancestral science and knowhow constitute the main aim of this study. A lot of vulnerable medicinal plants which are potentially effective against many dreadful diseases (Adjanohoun et al., 1996) could disappear even before the discovery of their medicinal proprieties (Diame, 2010).

The objective of this study is to analyse the traditional uses of the plants found in the Gribe rainforest (SouthEast Cameroon) among Baka pygmies as well as in the Kounabembé tribe. This study will also permit to assess the relative importance of medicinal plants within this Gribé locality. 


\section{Methods}

\subsection{Study area}

Gribé is a village located within the Yokadouma sub-division, in the Boumba and Ngoko Division of the East Region of Cameroon. It is situated between the latitude of $2^{\circ} 55^{\prime}$ and $3^{\circ} 20^{\prime}$ North and the East longitude of $14^{\circ} 45^{\prime}$ and $15^{\circ} 00^{\prime}$. The local population is about 700 inhabitants mainly made up of two ethnic groups of equal proportion (Toda, 2014) namely, the Baka pygmies who live on hunting and picking and the Kunabembé who are Bantu farmers (Tajeukem et al., 2014). Some non-indigenous populations like Vong-Vong, Biamgua and Kako also provided us with samples recipes of traditional medicine. Their main activities are hunting, fruit picking, subsistence fishing etc. There is no health facility in the area and schooling almost inexistent.

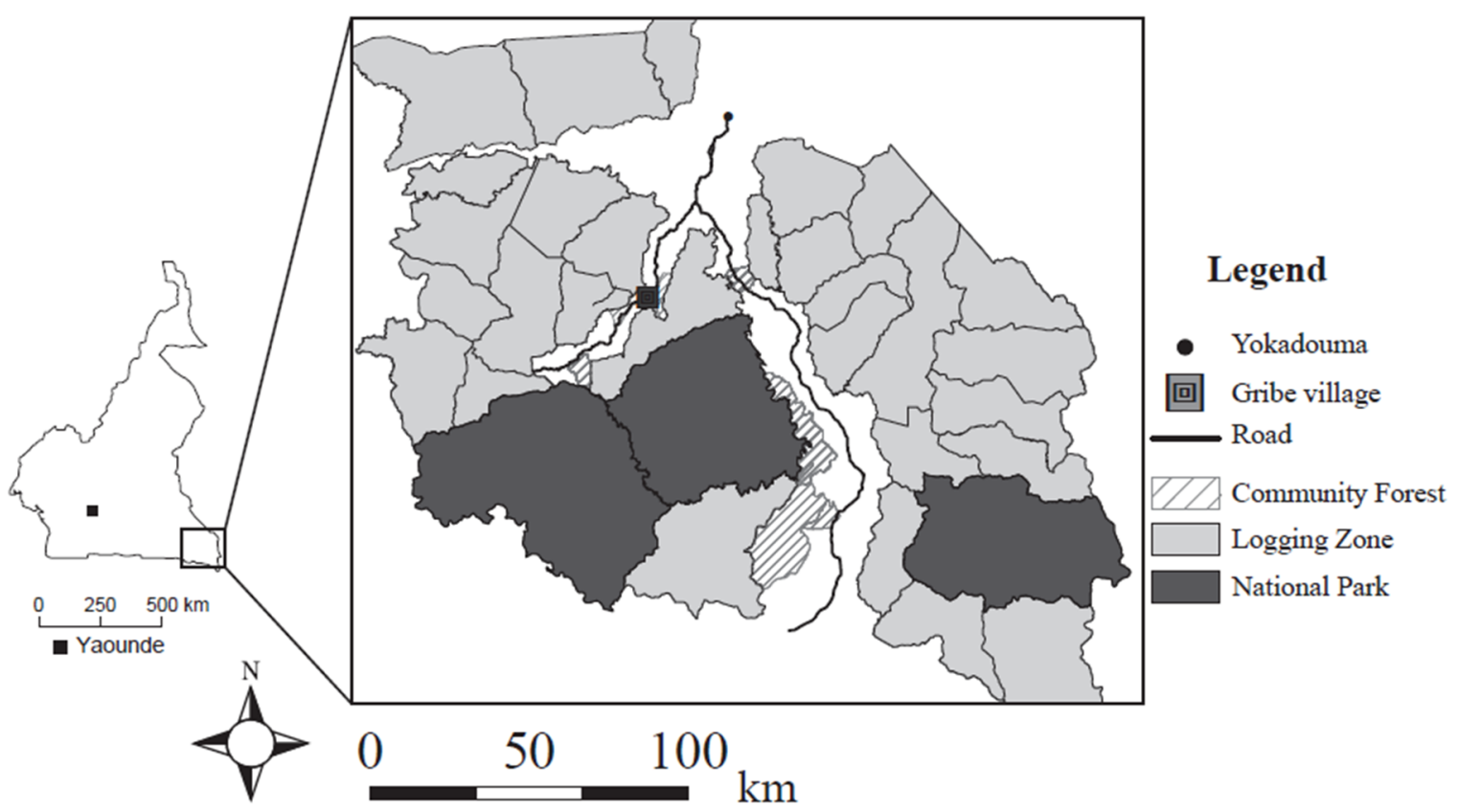

Figure 1. Location of the study area

The vegetation in this area is classified within the category of semi-deciduous forests dominated by Sterculiaceae and Ulmaceae (Letouzey, 1968; 1985; Nkongmeneck, 1999). The annual average of precipitations is ranged from $1200 \mathrm{~mm}$ to $1600 \mathrm{~mm}$ and the average annual temperature is about $24^{\circ} \mathrm{C}$ (Hirai, 2014). The widest river basin is that of the Boumba that has a width of about $200 \mathrm{~m}$, which makes it the biggest river in this region.

Studies carried out within the Gribé forest started since 2012, within the framework of the JICA/FOSAS cooperation project between Cameroon's government and Japan.

\subsection{Collection and data analysis}

The study was carried out in households and in many Baka settlements around the village of Gribé. Information was gathered through semi-structured interviews with people who resort to traditional medicine for primary healthcare (household traditional medicine). We relied on midwives, traditional healers, herbalists (expert of medicine or traditional healers medicine) and were inspired by methods described by Betti (1996; 2004), Nkongmeneck (1999), Lejoly (1993). Interviews that were conducted using the focus group technique with other members of the community enabled us to complete our information. The advantage of the focus group technique is that users of plants easily give complementary information. The information sought by the interviewers was on certain species of plants which are not commonly known, all the details on the recipes, preparation methods, the administration, the dosage, as well as the methods of collection. We worked in collaboration with all the ethnic groups of the area, namely Baka, Kounabembe, Vong-Vong, Biamgua and Kako. Information on the diagnosis of diseases was made available through semi structured interviews with specialists of traditional medicine. Diseases were classified and gathered following an adaptation proposed by the World Health Organisation (WHO, 2012). We were also inspired by the classification made available by some authors and researchers such as Betti \& Lejoly (2010), Adjanohoun et al. (1996) and Werner (2002). Other terms were adapted locally from the descriptions made through interviews.

The questionnaire was taken from the AFLORA database (African Flora) which is a data base on "the Traditional Use of Plants in Africa". The interview forms that were used served as sources of information on the fragments or organs of the exploited medicinal plants, their collection techniques, the preparation methods, the diseases treated and the dosage of the drugs given to patients. 
Plants samples were collected and identified with the collaboration of botanists from the Faculty of Science of the University of Yaoundé I, the National Institute of Medical research and Medicinal Plant Studies (NIMRMPS), the Millennium Ecological Museum and the Cameroon National Herbarium. Some manuals enabled us to identify certain species on the field.

\subsection{Data analysis}

Medicinal plants used in the locality of Gribé were counted and the frequencies calculated using the Excel base in order to assess the frequency of utilisation of each plant. The performance indexes of medicinal plants were calculated according to the method proposed by Betti (2004). The performance index establishes the relation between a specific flora and a global flora as well as the importance of a specific plant in the treatment of a given disease. The difference (D) between two proportions is thus used to define the performance index (Ip) which ranges from 0 to 3, following the arbitrary scale below:

- If $\mathrm{P}_{1}-\mathrm{P}_{2}<0, I p=0$, the plant is rejected and considered non-significant;

- If $0<\mathrm{P}_{1}-\mathrm{P}_{2} \leq 1 / 3$, Ip $=1$, average performance;

- If $1 / 3<\mathrm{P}_{1}-\mathrm{P}_{2} \leq 2 / 3$, Ip $=2$, high performance;

- If $\mathrm{P}_{1}-\mathrm{P}_{2}>2 / 3, I p=3$, very high performance.

$\mathrm{C}_{1}$ stands for the number of times the plant is mentioned in the treatment of a given disease. $\mathrm{C}_{2}$ is the number of times the plant is mentioned in a global list (its use in the treatment of all diseases). $\mathrm{C}_{3}$ stands for the total number of times the plant is mentioned for a given disease. $\mathrm{C}_{4}$ stands for the total number of times the plant is mentioned for all diseases. $\mathrm{P}_{1}$ (observed) and $\mathrm{P}_{2}$ (theoretical) are calculated as follows:

$\mathrm{P}_{1}=\mathrm{C}_{1} / \mathrm{C}_{2}$ and $\mathrm{P}_{2}=\mathrm{C}_{3} / \mathrm{C}_{4} ; \mathrm{D}=\mathrm{P}_{1}-\mathrm{P}_{2}$. The value of $\mathrm{D}$ enables to determine $I p$.

\section{Results}

\subsection{Analyses of traditional uses of plants}

A total of 240 informants were interviewed. Amongst that, we interviewed 192 men among whom there were no non-indigenes. However, among the 48 women interviewed, 44 were natives of the locality and 4 were nonindigenes (table 1). Furthermore, the Baka pygmies are the most represented in terms of numbers (165 against 71 Kounabembe, 02 Biamgua, 01 Vong-Vong and 01 Kako) (figure 2).

Table 1: Distribution per sex of the contribution of the local population in the use of NTFPs

\begin{tabular}{|l|l|l|l|}
\hline Sex & Origins & Numbers & Percentage (\%) \\
\hline \multirow{2}{*}{ Men } & Natives & 192 & 80 \\
\cline { 2 - 4 } & Non indigenous & 0 & 0 \\
\hline \multirow{2}{*}{ Women } & Natives & 44 & 18,33 \\
\cline { 2 - 4 } & Non indigenous & 4 & 1,67 \\
\hline Total & & $\mathbf{2 4 0}$ & $\mathbf{1 0 0}$ \\
\hline
\end{tabular}

Results in table1 shows that men made more proposals on the uses of plants in traditional pharmacopeia than women, making a ratio of $80 \%$ as against $20 \%$ for women. Consequently, the women are mostly left out when it comes to the use of plants in primary healthcare within the rural milieu.

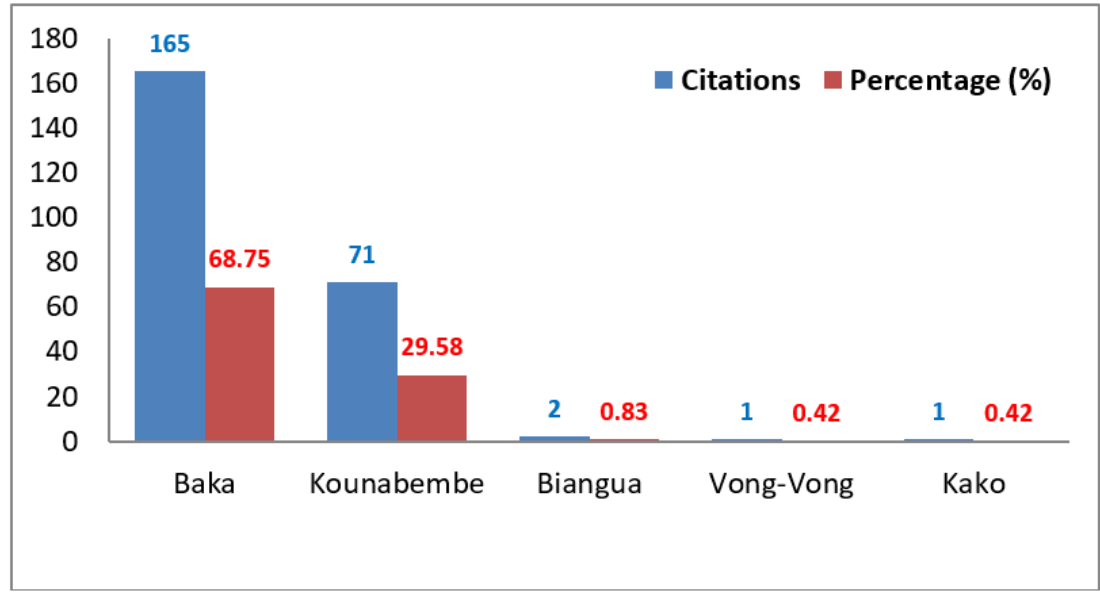

Figure 2. Relative importance of resource persons

Figure 2 shows that Baka populations of Gribé are more learned in the use of plants in traditional medicine in primary healthcare, thus confirming their close relationship with the forest. The great disparity in the figures is due to the fact that the Vong-Vong, the Biamgua and the Kako populations are not well represented in the village. Besides, the Kounabembe (71 people as against 165 for the Baka) have less experience in traditional 
pharmacopoeia than the Baka who have a great potential in that domain.

The most frequent diseases or symptoms of disease found in the locality are by order of importance sexual impotence, cough, backache, general tiredness, abscesses, lower abdomen affections for the women and others (fig 4). All these diseases affect the systems of the body such the digestive, the reproductive, the respiratory, gland and muscular systems etc.

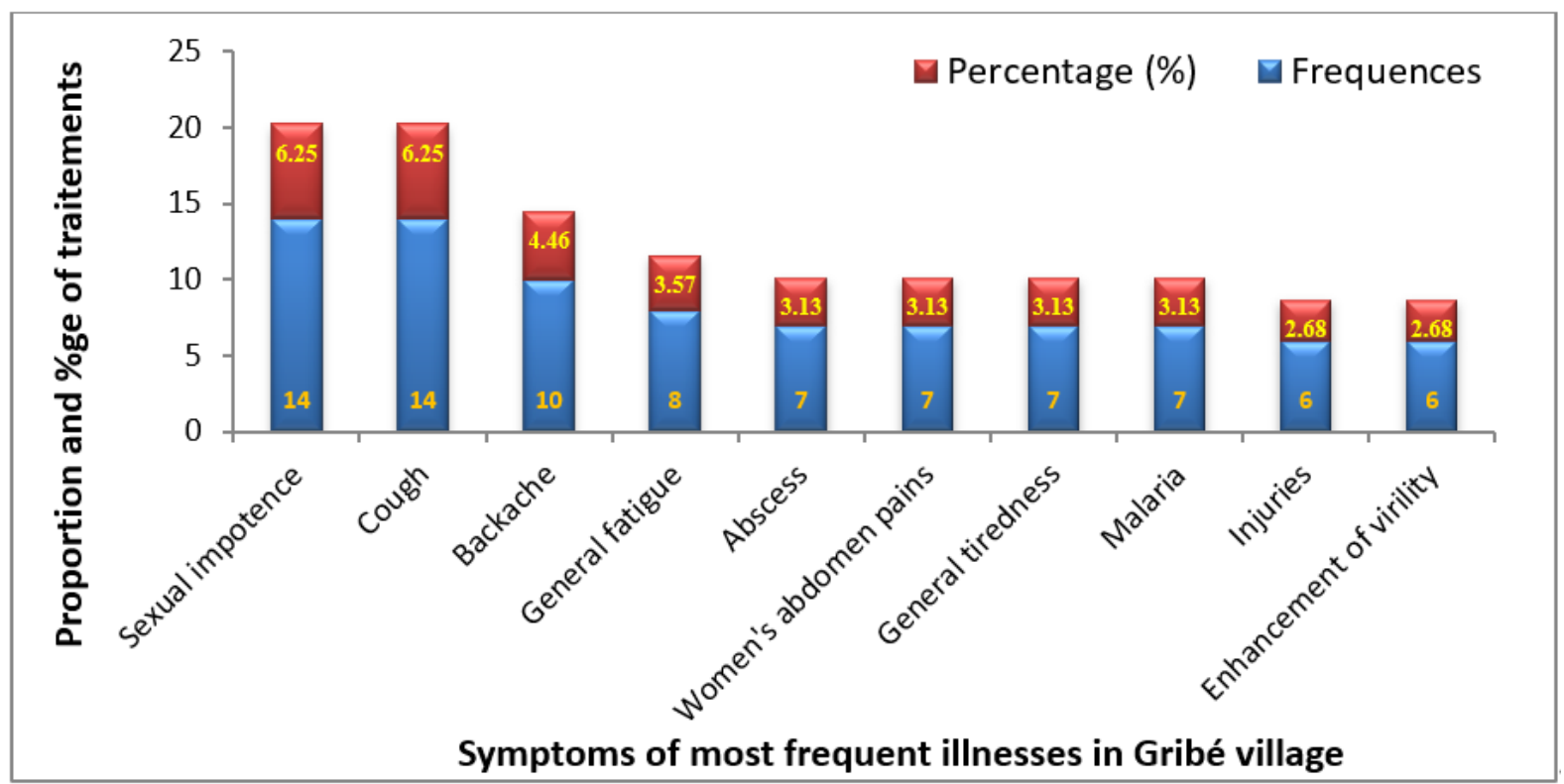

Figure 3. Relative importance of disease groups found in Gribé village

Diseases such as sexual impotence (14) representing 6.25\%, cough (14) for 6.25\%, backache (10) for 4.46\%, general fatigue (8) for $3.57 \%$, the affection of the lower abdomen for the women, general malaise and malaria (7) for $3.13 \%$ each other affections such as injuries, as well as the enhancement of virility have a frequency of 6 , that is $2.68 \%$.

The plants used for the treatment of diseases affecting the respiratory tract are among other: Breviea leptosperma, Calpocalyx sp., Coelocaryon preussii, Discoglypremna caloneura, Duboscia macrocarpa, Erybroma oblongum, Ficus exasperata, Ficus mucuso, Klainedoxa gabonensis, Mallotus oppositifolius, Mussaenda sp., Omphalocarpum lecomteanum, Pachypodanthium staudtii, Ricinodendron heudelotii with each species being mentioned at least once. Plants proposed as aphrodisiacs are: Celtis milbraedii (01 mention), Cola acuminate (01), Cola altissima (01), Diospyros crassiflora (01), Garcinia kola (02), Mildbraediodendron excelsum (01), Milicia excelsa (01), Ricinodendron heudelotii (01), Swartzia fistuloides (01), Triplochiton scleroxylon (01), Uapaca guineensis (01).

Other diseases such as bad spells, internal abscess, anti-abortive, the enhancement of sexual virility, the swelling of the abdomen, cancinogenic sores, mystical sores, tetanic sores and many others have only one proposed treatment.

Some plants are used more than others as well as for other purposes. 


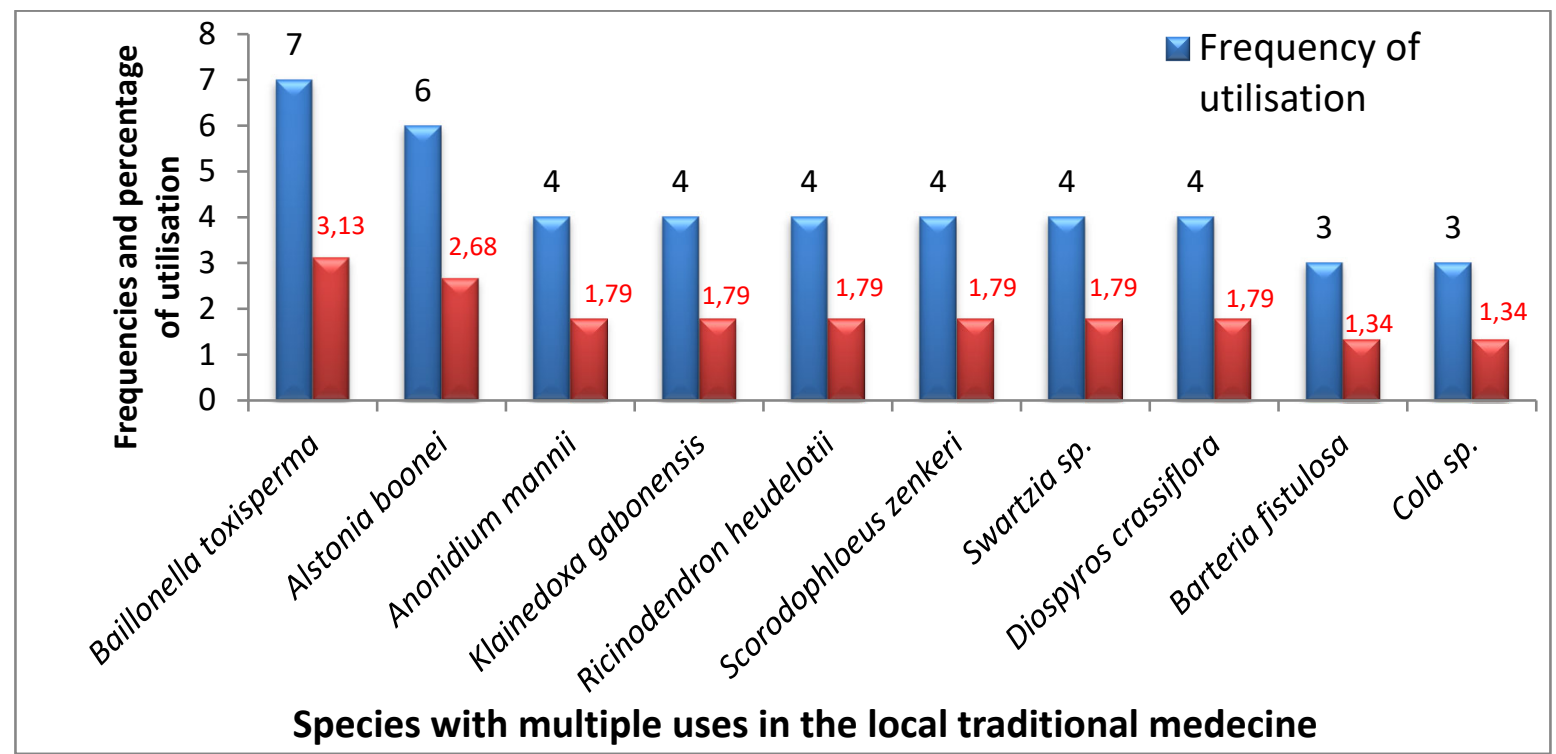

Species with multiple uses in the local traditional medecine

Some plants appear in many recipes: it is the case with Baillonella toxisperma that has seven therapeutic indicators (lower abdomen affection in women, sexual impotence, fever, infertility, backache, gastric disorders and lice). It is also the case with Alstonia boonei (swelling of the stomach, gonorrhea, malaria, jaundice, tuberculosis, intestinal worms) Anonidium mannii (gonorrhea, stimulation of milk secretion, infertility, syphilis) Klainedoxa ganonensis (gonorrhea, internal wounds, cough, HIV). Ricinodendron heudelotii (sexual impotence, aphrodisiac, stimulation of milk secretion, cough). Scorodophloeus zenkeri (abscess, backache, headache, intestinal worms). Swartzia sp. (general fatigue, hernia, kidney disorder, enhancement of virility). Diospyros crassifolia (lower abdominal pain, sexual impotence, painful menstruation, backache). Barteria fistulosa (antidote against poison, rheumatism, stimulation of breast secretion) and Cola sp. (madness, headache, malaria). We observe that 59 out of the 132 species of plants that we harvested are used to cure at least 2 diseases or 2 preparations, which is $66.41 \%$ of all the preparations. 73 plants out of 132 have a sole indication in the preparation of medicines, representing $32.59 \%$ of the proposed preparations. Other plants are used to treat many diseases in the locality. It is the case with Afrostyrax lepidiphyllus, Allanblackia floribunda, Celtis milbraedii, Bridelia grandis, Coelocaryon preussii, Beilshmiedia obscura, Cleistopholis patens, Lepidobotrys staudtii etc. This ratio is very representative given the number of species.

In the locality of Gribé, all parts of plants are used as medicines according to their accessibility, availability and efficiency in the treatment of diseases. We have registered some parts of plants used by the Baka and Kounabembe to extract medicine for auto medication for patients who come from all over the country. The table below shows the proportion and the used frequency of each part of the solicited plant for the extraction of medicine.

Table 2. Different percentages and the frequencies of the plant parts used.

\begin{tabular}{|r|l|r|r|}
\hline $\mathbf{N}^{\circ}$ & Parts used & Frequency & Percentage (\%) \\
\hline $\mathbf{1}$ & Bark & 164 & 73.21 \\
\hline $\mathbf{2}$ & Leaves & 20 & 8.93 \\
\hline $\mathbf{3}$ & Grains & 8 & 3.57 \\
\hline $\mathbf{4}$ & Sap & 6 & 2.68 \\
\hline $\mathbf{5}$ & Marrow of the bark & 6 & 2.68 \\
\hline $\mathbf{6}$ & Marrow of the stem & 6 & 2.68 \\
\hline $\mathbf{7}$ & Branches & 4 & 1.79 \\
\hline $\mathbf{8}$ & Fruit & 2 & 0.89 \\
\hline $\mathbf{9}$ & Honey of the nectar & 2 & 0.89 \\
\hline $\mathbf{1 0}$ & Rootwood & 1 & 0.45 \\
\hline $\mathbf{1 1}$ & Water of the barks & 1 & 0.45 \\
\hline $\mathbf{1 2}$ & Barks of roots & 1 & 0.45 \\
\hline $\mathbf{1 3}$ & Ants & 1 & 0.45 \\
\hline $\mathbf{1 4}$ & Nectar of flowers & 1 & 0.45 \\
\hline $\mathbf{1 5}$ & Roots & 1 & 0.45 \\
\hline
\end{tabular}

The part of plant mostly used is the bark of the stem which represents $73.21 \%$ of the parts of the plants on which the healing products are taken. Then come the leaves $(8.93 \%)$; the grains $(3.57 \%)$, the sap $(2.68 \%)$. The other parts are slightly represented within the proportions of the treatment of diseases that $0.45 \%$ for the roots and 
other parts.

Figure 5 below describes the various forms of preparation which are decoction, raw extraction, oral consumption, grinding, infusion, burning, trituration, heating, concoction and maceration. It also shows the different frequencies and percentages of the utilization.

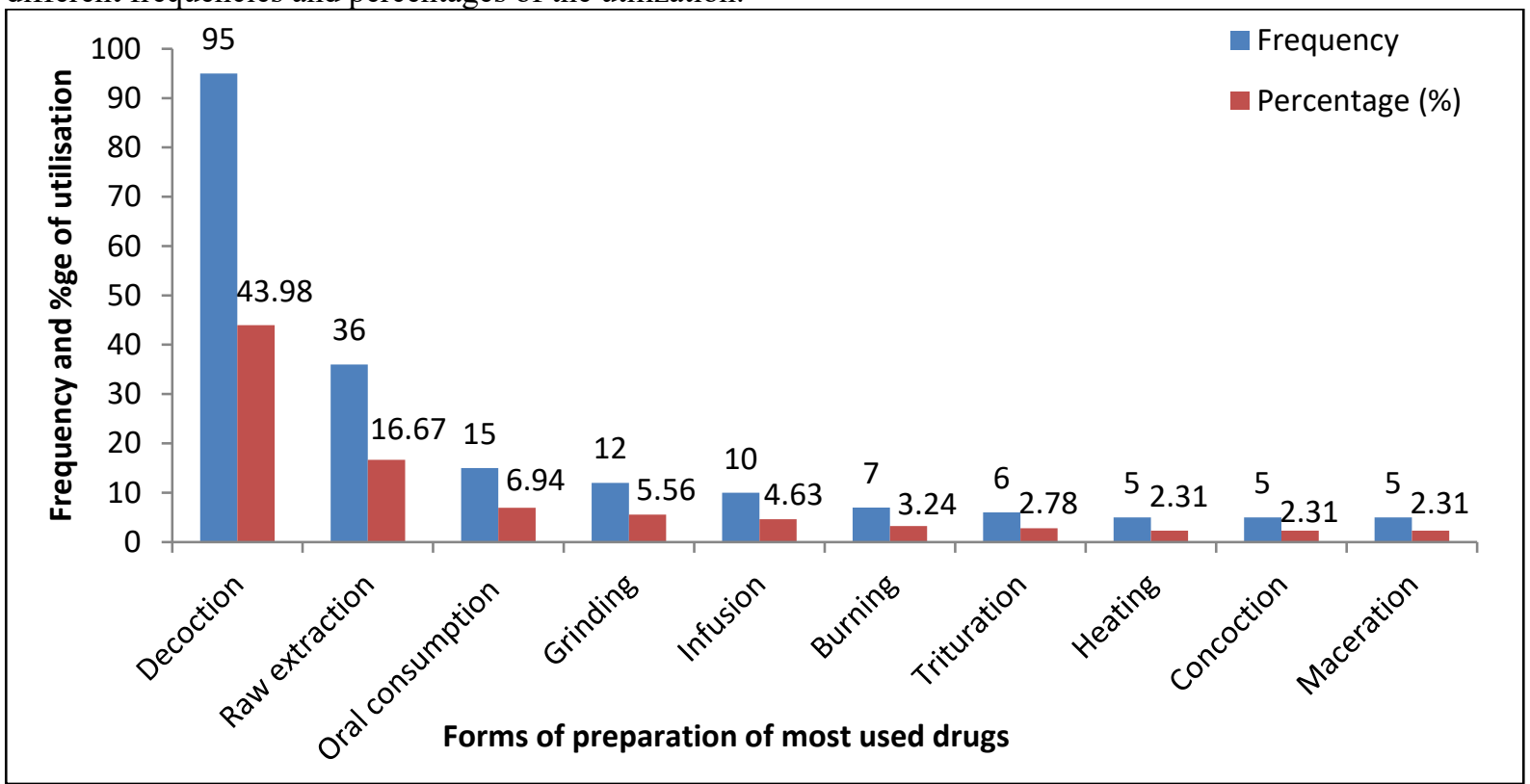

Figure 5. Various forms of preparation of the most used drugs

The forms of preparation of medicines are among others decoction, raw extraction, oral consumption, grinding, burning, triturating, heating on fire, maceration, infusion, grating, of the bark, sun drying etc. The most common forms of preparation are decoction (43.98 \%), raw extraction (16.66 \%), and oral consumption (6.94 \%). The drugs are administered through the oral, anal, skin, track, instillation, inhalation and scarification, etc. The figure below shows the different forms of consumption of drugs in the locality.

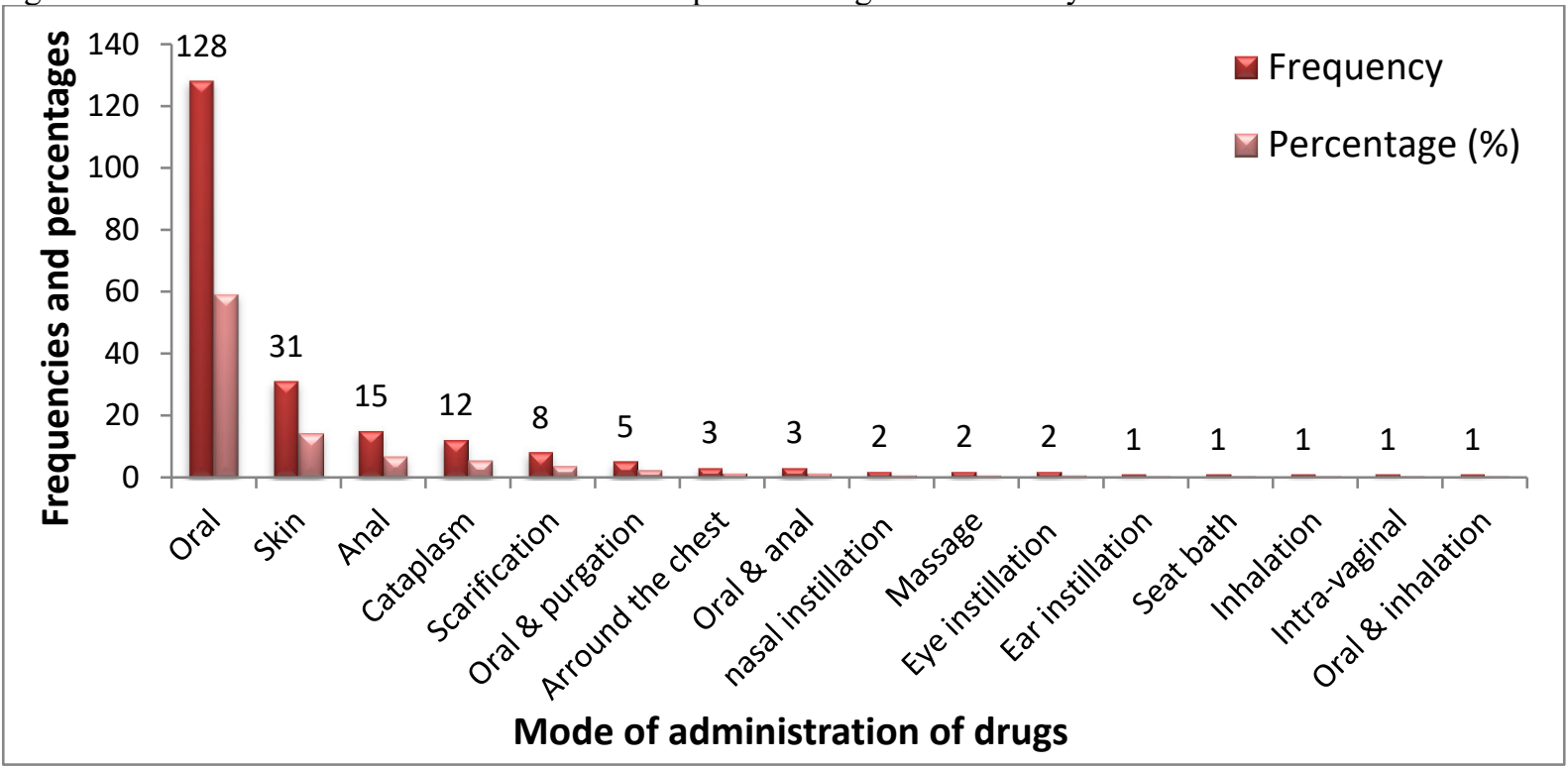

Figure 6. Different methods of administration of drugs in the Gribé village

It emerges from this table that the oral track is the most used in this locality, with a frequency of 128 which represents $59.25 \%$, followed by the skin (31) with $3.7 \%$; the anal track (15) with $6.94 \%$, the poultice (12) with $5.55 \%$, the scarification (8) with $3.7 \%$. These frequencies diminish as we move from nasal instillation, massage, eye instillation, ear instillation, to the seat bath and inhalation that has frequency of only 1 for $0.46 \%$. Some methods of administration are combined when prescribing the drugs to the patients.

\subsection{Relative importance of plants in the study area}

Table 3 below shows the highest performance indexes $(I p)$ of the study area of some specific diseases of Gribé 
village. The other performance indexes are found in document and they give the performance of each plant with regards to a given illness given the fact that we have in total 224 citations. It emerges from this table that 80 citations have a very high-performance index $(I p=3)$ for specific illnesses which are namely abscesses, the improvement of breast secretion, gonorrhea, internal wounds, anti-abortive etc. These diseases which plants show a very high Ip represent $36.60 \%$ of all the other diseases or symptoms of diseases registered in that locality.

Table 3. List of some diseases with a very high-performance index (Ip)

\begin{tabular}{|c|c|c|c|c|c|c|c|c|c|}
\hline Diseases & Plants & C1 & $\mathrm{C2}$ & P1 & C3 & $\mathrm{C4}$ & $\mathbf{P 2}$ & P1-P2 & $I p$ \\
\hline \multirow{4}{*}{ Abscess } & Erythrophleum suaveolens & 1 & 1 & 1 & 7 & 224 & 0,03 & 0,97 & 3 \\
\hline & Lindackeria dentata & 1 & 1 & 1 & 7 & 224 & 0,03 & 0,97 & 3 \\
\hline & Ongokea gore & 1 & 1 & 1 & 7 & 224 & 0,03 & 0,97 & 3 \\
\hline & Petersianthus macrocarpus & 2 & 2 & 1 & 7 & 224 & 0,03 & 0,97 & 3 \\
\hline Internal abscess & Detarium macrocarpum & 1 & 1 & 1 & 1 & 224 & 0,00 & 1,00 & 3 \\
\hline Anti-abortive & Pausinystalia joyimbe & 1 & 1 & 1 & 2 & 224 & 0,01 & 0,99 & 3 \\
\hline \multirow[t]{2}{*}{ Internal wounds } & Donnella ubanguiensis & 2 & 2 & 1 & 5 & 224 & 0,02 & 0,98 & 3 \\
\hline & Tricoscypha acuminata & 1 & 1 & 1 & 5 & 224 & 0,02 & 0,98 & 3 \\
\hline Tetanised wounds & Cleistopholis patens & 1 & 1 & 1 & 1 & 224 & 0,00 & 1,00 & 3 \\
\hline Constipation & $\begin{array}{l}\text { Tetrorchidium } \\
\text { didymostemon }\end{array}$ & 1 & 1 & 1 & 1 & 224 & 0,00 & 1,00 & 3 \\
\hline $\begin{array}{l}\text { Vaginal } \\
\text { contraction }\end{array}$ & Ficus etrangulator & 1 & 1 & 1 & 1 & 224 & 0,00 & 1,00 & 3 \\
\hline Madness & Tessmania africana & 1 & 1 & 1 & 3 & 224 & 0,01 & 0,99 & 3 \\
\hline Diarrhoea & Celtis zenkeri & 1 & 1 & 1 & 1 & 224 & 0,00 & 1,00 & 3 \\
\hline Generalised pains & Lepidobotrys staudtii & 1 & 1 & 1 & 1 & 224 & 0,00 & 1,00 & 3 \\
\hline Muscular pains & Desplatia dewevrei & 1 & 1 & 1 & 1 & 224 & 0,00 & 1,00 & 3 \\
\hline Bloody dysentery & Xylopia hypolampra & 1 & 1 & 1 & 1 & 224 & 0,00 & 1,00 & 3 \\
\hline Sprain & Cola lateritia & 1 & 1 & 1 & 2 & 224 & 0,01 & 0,99 & 3 \\
\hline \multirow{3}{*}{ Sexual impotence } & Celtis milbraedii & 1 & 1 & 1 & 14 & 224 & 0,06 & 0,94 & 3 \\
\hline & Garcinia kola & 2 & 2 & 1 & 14 & 224 & 0,06 & 0,94 & 3 \\
\hline & Swartzia fistuloides & 1 & 1 & 1 & 14 & 224 & 0,06 & 0,94 & 3 \\
\hline \multirow{3}{*}{ General tiredness } & Cylicodiscus gabunensis & 2 & 2 & 1 & 8 & 224 & 0,04 & 0,96 & 3 \\
\hline & Santiria trimera & 1 & 1 & 1 & 8 & 224 & 0,04 & 0,96 & 3 \\
\hline & Strombosia pustulata & 1 & 1 & 1 & 8 & 224 & 0,04 & 0,96 & 3 \\
\hline Filariasies & Polyalthia suaveolens & 1 & 1 & 1 & 1 & 224 & 0,00 & 1,00 & 3 \\
\hline Scabies & Brenania brieyi & 2 & 2 & 1 & 1 & 224 & 0,00 & 1,00 & 3 \\
\hline Piles & Canthium sp. & 1 & 1 & 1 & 1 & 224 & 0,00 & 1,00 & 3 \\
\hline Hernia & Diospyros sp. & 1 & 1 & 1 & 6 & 224 & 0,03 & 0,97 & 3 \\
\hline Indigestion & Maesopsis eminii & 1 & 1 & 1 & 2 & 224 & 0,01 & 0,99 & 3 \\
\hline $\begin{array}{l}\text { Infertility } \\
\text { (women) }\end{array}$ & Neoboutonia glabrescens & 1 & 1 & 1 & 1 & 224 & 0,00 & 1,00 & 3 \\
\hline STIs & Tieghemella africana & 1 & 1 & 1 & 3 & 224 & 0,01 & 0,99 & 3 \\
\hline Jaundice & $\begin{array}{l}\text { Entandrophragma } \\
\text { cylindricum }\end{array}$ & 2 & 2 & 1 & 3 & 224 & 0,01 & 0,99 & 3 \\
\hline Ill luck & Afrostyrax lepidiphyllus & 1 & 1 & 1 & 1 & 224 & 0,00 & 1,00 & 3 \\
\hline \multirow{4}{*}{ Backache } & Copaifera mildbraedii & 1 & 1 & 1 & 12 & 224 & 0,05 & 0,95 & 3 \\
\hline & Lecaniodiscus $\mathrm{sp}$. & 1 & 1 & 1 & 12 & 224 & 0,05 & 0,95 & 3 \\
\hline & Leptonychia sp. & 1 & 1 & 1 & 12 & 224 & 0,05 & 0,95 & 3 \\
\hline & Tetrapleura tetraptera & 1 & 1 & 1 & 12 & 224 & 0,05 & 0,95 & 3 \\
\hline Chest pain & Homalium sp. & 1 & 1 & 1 & 3 & 224 & 0,01 & 0,99 & 3 \\
\hline Ribs pain & Amanoa bracteosa & 2 & 2 & 1 & 3 & 224 & 0,01 & 0,99 & 3 \\
\hline Mole disease & Nesogordonia papaverifera & 1 & 1 & 1 & 1 & 224 & 0,00 & 1,00 & 3 \\
\hline Infant diseases & Celtis philippendis & 1 & 1 & 1 & 1 & 224 & 0,00 & 1,00 & 3 \\
\hline \multirow{6}{*}{$\begin{array}{l}\text { General } \\
\text { discomfort }\end{array}$} & Amphimas pterocarpoides & 1 & 1 & 1 & 7 & 224 & 0,03 & 0,97 & 3 \\
\hline & Beilshmiedia obscura & 1 & 1 & 1 & 7 & 224 & 0,03 & 0,97 & 3 \\
\hline & Cordia platythyrsa & 1 & 1 & 1 & 7 & 224 & 0,03 & 0,97 & 3 \\
\hline & Entandrophragma candollei & 1 & 1 & 1 & 7 & 224 & 0,03 & 0,97 & 3 \\
\hline & Entandrophragma utile & 1 & 1 & 1 & 7 & 224 & 0,03 & 0,97 & 3 \\
\hline & Trichilia welwitschii & 1 & 1 & 1 & 7 & 224 & 0,03 & 0,97 & 3 \\
\hline
\end{tabular}




\begin{tabular}{|c|c|c|c|c|c|c|c|c|c|}
\hline Diseases & Plants & C1 & $\mathrm{C2}$ & P1 & $\mathbf{C 3}$ & C4 & $\mathbf{P 2}$ & P1-P2 & $I p$ \\
\hline Headaches & Beilshmiedia sp.2 & 1 & 1 & 1 & 4 & 224 & 0,02 & 0,98 & 3 \\
\hline \multirow{3}{*}{ Snake bite } & Rauvolfia caffra & 1 & 1 & 1 & 3 & 224 & 0,01 & 0,99 & 3 \\
\hline & Rauvolfia grandifolia & 1 & 1 & 1 & 3 & 224 & 0,01 & 0,99 & 3 \\
\hline & Rauvolfia macrophylla & 1 & 1 & 1 & 3 & 224 & 0,01 & 0,99 & 3 \\
\hline Hearth palpitation & Celtis tessmannii & 1 & 1 & 1 & 1 & 224 & 0,00 & 1,00 & 3 \\
\hline \multirow[t]{2}{*}{ Sexual virility } & Allanblackia floribunda & 2 & 2 & 1 & 6 & 224 & 0,03 & 0,97 & 3 \\
\hline & Allanblackia gabonensis & 1 & 1 & 1 & 6 & 224 & 0,03 & 0,97 & 3 \\
\hline \multirow[t]{2}{*}{ Rheumatism } & Dichaethanthera sp. & 2 & 2 & 1 & 3 & 224 & 0,01 & 0,99 & 3 \\
\hline & Gossypium sp. & 1 & 1 & 1 & 3 & 224 & 0,01 & 0,99 & 3 \\
\hline Measles & Drypetes gossweileri & 2 & 2 & 1 & 2 & 224 & 0,01 & 0,99 & 3 \\
\hline $\begin{array}{l}\text { Strengthening of } \\
\text { bones }\end{array}$ & Garcinia sp.1 & 1 & 1 & 1 & 1 & 224 & 0,00 & 1,00 & 3 \\
\hline \multirow{11}{*}{ Cough } & Breviea leptosperma & 1 & 1 & 1 & 14 & 224 & 0,06 & 0,94 & 3 \\
\hline & Calpocalyx sp. & 1 & 1 & 1 & 14 & 224 & 0,06 & 0,94 & 3 \\
\hline & Coelocaryon preussii & 1 & 1 & 1 & 14 & 224 & 0,06 & 0,94 & 3 \\
\hline & Discoglypremna caloneura & 1 & 1 & 1 & 14 & 224 & 0,06 & 0,94 & 3 \\
\hline & Duboscia macrocarpa & 1 & 1 & 1 & 14 & 224 & 0,06 & 0,94 & 3 \\
\hline & Erybroma oblongum & 1 & 1 & 1 & 14 & 224 & 0,06 & 0,94 & 3 \\
\hline & Mallotus oppositifolius & 1 & 1 & 1 & 14 & 224 & 0,06 & 0,94 & 3 \\
\hline & $\begin{array}{l}\text { Omphalocarpum } \\
\text { lecomteanum }\end{array}$ & 1 & 1 & 1 & 14 & 224 & 0,06 & 0,94 & 3 \\
\hline & Pachypodanthium staudtii & 1 & 1 & 1 & 14 & 224 & 0,06 & 0,94 & 3 \\
\hline & Anthocleista schweinfurtii & 1 & 1 & 1 & 1 & 224 & 0,00 & 1,00 & 3 \\
\hline & Musanga cecropioides & 1 & 1 & 1 & 1 & 224 & 0,00 & 1,00 & 3 \\
\hline \multirow[t]{2}{*}{ Intestinal worms } & Octolobus sp. & 1 & 1 & 1 & 5 & 224 & 0,02 & 0,98 & 3 \\
\hline & Trichilia heudelotii & 1 & 1 & 1 & 5 & 224 & 0,02 & 0,98 & 3 \\
\hline Bad luck & Irvingia gabonensis & 1 & 2 & 0,5 & 1 & 224 & 0 & 0,50 & 2 \\
\hline \multirow[t]{2}{*}{ Abscess } & Myrianthus arboreus & 2 & 3 & 0,7 & 7 & 224 & 0,03 & 0,64 & 2 \\
\hline & Pericopsis elata & 1 & 2 & 0,5 & 7 & 224 & 0,03 & 0,47 & 2 \\
\hline \multirow[t]{2}{*}{ Abortive } & Mansonia altissima & 1 & 2 & 0,5 & 2 & 224 & 0,01 & 0,49 & 2 \\
\hline & Pentaclethra macrophylla & 1 & 2 & 0,5 & 2 & 224 & 0,01 & 0,49 & 2 \\
\hline \multirow{5}{*}{ Injuries } & Dicranolepis sp. & 2 & 3 & 0,7 & 6 & 224 & 0,03 & 0,64 & 2 \\
\hline & Gnetum africanum & 1 & 2 & 0,5 & 6 & 224 & 0,03 & 0,47 & 2 \\
\hline & Gnetum buccholzianum & 1 & 2 & 0,5 & 6 & 224 & 0,03 & 0,47 & 2 \\
\hline & Staudtia kamerunensis & 1 & 2 & 0,5 & 6 & 224 & 0,03 & 0,47 & 2 \\
\hline & Tabernaemontana crassa & 1 & 2 & 0,5 & 6 & 224 & 0,03 & 0,47 & 2 \\
\hline Internal injuries & Sterculia tragacantha & 1 & 2 & 0,5 & 5 & 224 & 0,02 & 0,48 & 2 \\
\hline Mystical wounds & Fagara heitzii & 1 & 2 & 0,5 & 1 & 224 & 0 & 0,50 & 2 \\
\hline Fits of madness & Cola altissima & 1 & 2 & 0,5 & 3 & 224 & 0,01 & 0,49 & 2 \\
\hline Perforated teeth & Uapaca guineensis & 1 & 2 & 0,5 & 1 & 224 & 0 & 0,50 & 2 \\
\hline Dysentery & Picralima nitida & 1 & 2 & 0,5 & 1 & 224 & 0 & 0,50 & 2 \\
\hline Sprain & Trema orientalis & 1 & 2 & 0,5 & 2 & 224 & 0,01 & 0,49 & 2 \\
\hline $\begin{array}{l}\text { Facilitating } \\
\text { childbirth }\end{array}$ & Ficus exasperata & 1 & 2 & 0,5 & 14 & 224 & 0,06 & 0,44 & 2 \\
\hline \multirow{5}{*}{ Sexual weakness } & Milicia excelsa & 2 & 3 & 0,7 & 14 & 224 & 0,06 & 0,60 & 2 \\
\hline & Cola acuminata & 1 & 2 & 0,5 & 14 & 224 & 0,06 & 0,44 & 2 \\
\hline & Cola altissima & 1 & 2 & 0,5 & 14 & 224 & 0,06 & 0,44 & 2 \\
\hline & $\begin{array}{l}\text { Mildbraediodendron } \\
\text { excelsum }\end{array}$ & 1 & 2 & 0,5 & 14 & 224 & 0,06 & 0,44 & 2 \\
\hline & Uapaca guineensis & 1 & 2 & 0,5 & 14 & 224 & 0,06 & 0,44 & 2 \\
\hline \multirow[t]{2}{*}{ General tiredness } & Rauvolfia vomitoria & 1 & 2 & 0,5 & 8 & 224 & 0,04 & 0,46 & 2 \\
\hline & Staudtia kamerunensis & 1 & 2 & 0,5 & 8 & 224 & 0,04 & 0,46 & 2 \\
\hline \multirow{3}{*}{ Hernia } & Meicarpidium lepidotum & 1 & 2 & 0,5 & 6 & 224 & 0,03 & 0,47 & 2 \\
\hline & Millettia sanagana & 1 & 2 & 0,5 & 6 & 224 & 0,03 & 0,47 & 2 \\
\hline & Rauvolfia vomitoria & 1 & 2 & 0,5 & 6 & 224 & 0,03 & 0,47 & 2 \\
\hline Hypertension & Angylocalyx pynaertii & 1 & 2 & 0,5 & 1 & 224 & 0 & 0,50 & 2 \\
\hline
\end{tabular}




\begin{tabular}{|c|c|c|c|c|c|c|c|c|c|}
\hline Diseases & Plants & C1 & $\mathrm{C2}$ & P1 & $\mathbf{C 3}$ & $\mathrm{C4}$ & $\mathbf{P 2}$ & P1-P2 & $I p$ \\
\hline Indigestion & Gambeya lacourtiana & 1 & 2 & 0,5 & 2 & 224 & 0,01 & 0,49 & 2 \\
\hline $\begin{array}{l}\text { Testicular } \\
\text { inflammation }\end{array}$ & Tabernaemontana crassa & 1 & 2 & 0,5 & 1 & 224 & 0 & 0,50 & 2 \\
\hline STI & Pentaclethra macrophylla & 1 & 2 & 0,5 & 3 & 224 & 0,01 & 0,49 & 2 \\
\hline Jaundice & Ceiba pentandra & 1 & 2 & 0,5 & 3 & 224 & 0,01 & 0,49 & 2 \\
\hline \multirow{3}{*}{ Back pain } & Cylicodiscus gabunensis & 1 & 2 & 0,5 & 12 & 224 & 0,05 & 0,45 & 2 \\
\hline & Gambeya lacourtiana & 1 & 2 & 0,5 & 12 & 224 & 0,05 & 0,45 & 2 \\
\hline & Mansonia altissima & 1 & 2 & 0,5 & 12 & 224 & 0,05 & 0,45 & 2 \\
\hline Evil eye & $\begin{array}{l}\text { Entandrophragma } \\
\text { cylindricum }\end{array}$ & 1 & 2 & 0,5 & 12 & 224 & 0,05 & 0,45 & 2 \\
\hline \multirow[t]{2}{*}{ Sore Chest } & Celtis adolfi-friderici & 1 & 2 & 0,5 & 3 & 224 & 0,01 & 0,49 & 2 \\
\hline & Sterculia tragacantha & 1 & 2 & 0,5 & 3 & 224 & 0,01 & 0,49 & 2 \\
\hline General malaise & Amanoa bracteosa & 1 & 2 & 0,5 & 7 & 224 & 0,03 & 0,47 & 2 \\
\hline Headache & Fagara heitzii & 1 & 2 & 0,5 & 4 & 224 & 0,02 & 0,48 & 2 \\
\hline \multirow{8}{*}{ Stomachache } & Bridelia grandis & 1 & 1 & 1 & 2 & 224 & 0,01 & 0,99 & 3 \\
\hline & Hallea stipulosa & 1 & 1 & 1 & 2 & 224 & 0,01 & 0,99 & 3 \\
\hline & Mussaenda sp. & 1 & 2 & 0,5 & 4 & 224 & 0,02 & 0,48 & 2 \\
\hline & Synsepalum aubrevillei & 1 & 1 & 1 & 4 & 224 & 0,02 & 0,98 & 3 \\
\hline & Ficus sp. & 1 & 1 & 1 & 4 & 224 & 0,02 & 0,98 & 3 \\
\hline & Psychotria sp.1 & 1 & 1 & 1 & 4 & 224 & 0,02 & 0,98 & 3 \\
\hline & $\begin{array}{l}\text { Distemonanthus } \\
\text { benthamiana }\end{array}$ & 1 & 2 & 0,5 & 1 & 224 & 0 & 0,50 & 2 \\
\hline & Ochthocosmus africanus & 1 & 2 & 0,5 & 4 & 224 & 0,02 & 0,48 & 2 \\
\hline Purgative & Garcinia puntata & 1 & 2 & 0,5 & 1 & 224 & 0 & 0,50 & 2 \\
\hline Painful periods & Meicarpidium lepidotum & 1 & 2 & 0,5 & 2 & 224 & 0,01 & 0,49 & 2 \\
\hline \multirow{2}{*}{$\begin{array}{l}\text { Regulation of } \\
\text { prostate }\end{array}$} & Gnetum africanum & 1 & 2 & 0,5 & 2 & 224 & 0,01 & 0,49 & 2 \\
\hline & Gnetum buccholzianum & 1 & 2 & 0,5 & 2 & 224 & 0,01 & 0,49 & 2 \\
\hline $\begin{array}{l}\text { Regulating milk } \\
\text { secretion }\end{array}$ & Terminalia superba & 2 & 3 & 0,7 & 1 & 224 & 0 & 0,66 & 2 \\
\hline \multirow{3}{*}{$\begin{array}{l}\text { Strengthening } \\
\text { sexual virility }\end{array}$} & Celtis adolfi-friderici & 1 & 2 & 0,5 & 6 & 224 & 0,03 & 0,47 & 2 \\
\hline & $\begin{array}{l}\text { Mildbraediodendron } \\
\text { excelsum }\end{array}$ & 1 & 2 & 0,5 & 6 & 224 & 0,03 & 0,47 & 2 \\
\hline & Ochthocosmus africanus & 1 & 2 & 0,5 & 6 & 224 & 0,03 & 0,47 & 2 \\
\hline Tetanus antitoxin & Pausinystalia joyimbe & 1 & 2 & 0,5 & 1 & 224 & 0 & 0,50 & 2 \\
\hline Sexual stimulant & Millettia sanagana & 1 & 2 & 0,5 & 1 & 224 & 0 & 0,50 & 2 \\
\hline $\begin{array}{l}\text { Stimulation of } \\
\text { hunger in babies }\end{array}$ & Trema orientalis & 1 & 2 & 0,5 & 4 & 224 & 0,02 & 0,48 & 2 \\
\hline $\begin{array}{l}\text { Support } \\
\text { convalescent }\end{array}$ & Albizia glaberrima & 1 & 2 & 0,5 & 1 & 224 & 0 & 0,50 & 2 \\
\hline Deafness & Ceiba pentandra & 1 & 2 & 0,5 & 1 & 224 & 0 & 0,50 & 2 \\
\hline \multirow[t]{2}{*}{ Cough } & Ficus exasperata & 1 & 2 & 0,5 & 14 & 224 & 0,06 & 0,44 & 2 \\
\hline & Mussaenda sp. & 1 & 2 & 0,5 & 14 & 224 & 0,06 & 0,44 & 2 \\
\hline Smallpox & Drypetes gossweileri & 1 & 2 & 0,5 & 1 & 224 & 0 & 0,50 & 2 \\
\hline \multirow[t]{2}{*}{ Emetic } & Garcinia puntata & 1 & 2 & 0,5 & 2 & 224 & 0,01 & 0,49 & 2 \\
\hline & Pericopsis elata & 1 & 2 & 0,5 & 2 & 224 & 0,01 & 0,49 & 2 \\
\hline Abscess & Scorodophloeus zenkeri & 1 & 4 & 0,3 & 7 & 224 & 0,03 & 0,22 & 1 \\
\hline \multirow{4}{*}{$\begin{array}{l}\text { Stimulating milk } \\
\text { secretion }\end{array}$} & Pachyelasma tessmannii & 1 & 1 & 1 & 3 & 224 & 0,01 & 0,99 & 3 \\
\hline & Symphonia globulifera & 1 & 1 & 1 & 3 & 224 & 0,01 & 0,99 & 3 \\
\hline & Barteria fistulosa & 1 & 3 & 0,3 & 3 & 224 & 0,01 & 0,32 & 1 \\
\hline & Irvingia gabonensis & 1 & 2 & 0,5 & 4 & 224 & 0,02 & 0,48 & 2 \\
\hline $\begin{array}{l}\text { Improvement of } \\
\text { taste baby milk }\end{array}$ & Hunteria umbellata & 1 & 3 & 0,3 & 3 & 224 & 0,01 & 0,32 & 1 \\
\hline \multirow[t]{4}{*}{ Bloated belly } & Alstonia boonei & 1 & 6 & 0,2 & 1 & 224 & 0 & 0,16 & 1 \\
\hline & Baillonella toxisperma & 1 & 7 & 0,1 & 7 & 224 & 0,03 & 0,11 & 1 \\
\hline & Diospyros crassiflora & 1 & 4 & 0,3 & 7 & 224 & 0,03 & 0,22 & 1 \\
\hline & Margaritaria discoidea & 1 & 3 & 0,3 & 7 & 224 & 0,03 & 0,30 & 1 \\
\hline
\end{tabular}




\begin{tabular}{|c|c|c|c|c|c|c|c|c|c|}
\hline Diseases & Plants & C1 & $\mathbf{C 2}$ & P1 & $\mathbf{C 3}$ & $\mathrm{C4}$ & $\mathbf{P 2}$ & P1-P2 & $I p$ \\
\hline \multirow{4}{*}{$\begin{array}{l}\text { Lower abdomen } \\
\text { pains of women }\end{array}$} & Albizia glaberrima & 1 & 2 & 0,5 & 7 & 224 & 0,03 & 0,47 & 2 \\
\hline & Cola acuminata & 1 & 2 & 0,5 & 7 & 224 & 0,03 & 0,47 & 2 \\
\hline & Diopyros abissinica & 1 & 2 & 0,5 & 7 & 224 & 0,03 & 0,47 & 2 \\
\hline & $\begin{array}{l}\text { Distemonanthus } \\
\text { benthamiana }\end{array}$ & 1 & 2 & 0,5 & 7 & 224 & 0,03 & 0,47 & 2 \\
\hline \multirow{5}{*}{ Gonorrhea } & Angylocalyx pynaertii & 2 & 2 & 1 & 5 & 224 & 0,02 & 0,98 & 3 \\
\hline & Anonidium mannii & 1 & 4 & 0,3 & 5 & 224 & 0,02 & 0,23 & 1 \\
\hline & Klainedoxa gabonensis & 1 & 4 & 0,3 & 5 & 224 & 0,02 & 0,23 & 1 \\
\hline & Raphia regalis & 1 & 3 & 0,3 & 5 & 224 & 0,02 & 0,31 & 1 \\
\hline & Alstonia boonei & 1 & 6 & 0,2 & 5 & 224 & 0,02 & 0,14 & 1 \\
\hline Injuries & Corynthe pachyceras & 1 & 3 & 0,3 & 6 & 224 & 0,03 & 0,31 & 1 \\
\hline $\begin{array}{l}\text { Carcinogenic } \\
\text { injuries }\end{array}$ & Margaritaria discoidea & 1 & 3 & 0,3 & 1 & 224 & 0 & 0,33 & 1 \\
\hline \multirow[t]{2}{*}{ Internal injuries } & Corynthe pachyceras & 1 & 3 & 0,3 & 5 & 224 & 0,02 & 0,31 & 1 \\
\hline & Klainedoxa gabonensis & 1 & 4 & 0,3 & 5 & 224 & 0,02 & 0,23 & 1 \\
\hline Burn & Raphia regalis & 1 & 3 & 0,3 & 1 & 224 & 0 & 0,33 & 1 \\
\hline Against poison & Barteria fistulosa & 1 & 3 & 0,3 & 1 & 224 & 0 & 0,33 & 1 \\
\hline Fits of madness & Cola sp. & 1 & 3 & 0,3 & 3 & 224 & 0,01 & 0,32 & 1 \\
\hline \multirow{4}{*}{ Sexual weakness } & Baillonella toxisperma & 1 & 7 & 0,1 & 14 & 224 & 0,06 & 0,08 & 1 \\
\hline & Diospyros crassiflora & 1 & 4 & 0,3 & 14 & 224 & 0,06 & 0,19 & 1 \\
\hline & Ricinodendron heudelotii & 1 & 4 & 0,3 & 14 & 224 & 0,06 & 0,19 & 1 \\
\hline & Triplochiton scleroxylon & 1 & 3 & 0,3 & 14 & 224 & 0,06 & 0,27 & 1 \\
\hline \multirow{3}{*}{ General tiredness } & Corynthe pachyceras & 1 & 3 & 0,3 & 8 & 224 & 0,04 & 0,30 & 1 \\
\hline & Swartzia $\mathrm{sp}$ & 1 & 4 & 0,3 & 8 & 224 & 0,04 & 0,21 & 1 \\
\hline & Triplochiton scleroxylon & 1 & 3 & 0,3 & 8 & 224 & 0,04 & 0,30 & 1 \\
\hline Fever & Baillonella toxisperma & 1 & 7 & 0,1 & 1 & 224 & 0 & 0,14 & 1 \\
\hline Invigorating & Ricinodendron heudelotii & 1 & 4 & 0,3 & 1 & 224 & 0 & 0,25 & 1 \\
\hline \multirow[t]{2}{*}{ Hernia } & Swartzia sp. & 1 & 4 & 0,3 & 6 & 224 & 0,03 & 0,22 & 1 \\
\hline & Terminalia superba & 1 & 3 & 0,3 & 6 & 224 & 0,03 & 0,31 & 1 \\
\hline $\mathrm{HIV}$ & Klainedoxa gabonensis & 1 & 4 & 0,3 & 1 & 224 & 0 & 0,25 & 1 \\
\hline \multirow[t]{2}{*}{ Infertility } & Anonidium mannii & 1 & 4 & 0,3 & 2 & 224 & 0,01 & 0,24 & 1 \\
\hline & Baillonella toxisperma & 1 & 7 & 0,1 & 2 & 224 & 0,01 & 0,13 & 1 \\
\hline STI & Ficus тисияо & 1 & 3 & 0,3 & 3 & 224 & 0,01 & 0,32 & 1 \\
\hline Jaundice & Alstonia boonei & 1 & 6 & 0,2 & 3 & 224 & 0,01 & 0,15 & 1 \\
\hline Tooth ache & Panda oleosa & 1 & 3 & 0,3 & 1 & 224 & 0 & 0,33 & 1 \\
\hline \multirow{4}{*}{ Back pain } & Baillonella toxisperma & 2 & 7 & 0,3 & 12 & 224 & 0,05 & 0,23 & 1 \\
\hline & Diospyros crassiflora & 1 & 4 & 0,3 & 12 & 224 & 0,05 & 0,20 & 1 \\
\hline & Scorodophloeus zenkeri & 1 & 4 & 0,3 & 12 & 224 & 0,05 & 0,20 & 1 \\
\hline & Hunteria umbellata & 1 & 3 & 0,3 & 12 & 224 & 0,05 & 0,28 & 1 \\
\hline \multirow{3}{*}{ Kidney failure } & Margaritaria discoidea & 1 & 3 & 0,3 & 3 & 224 & 0,01 & 0,32 & 1 \\
\hline & Panda oleosa & 1 & 3 & 0,3 & 3 & 224 & 0,01 & 0,32 & 1 \\
\hline & Swartzia sp. & 1 & 4 & 0,3 & 3 & 224 & 0,01 & 0,24 & 1 \\
\hline \multirow[t]{2}{*}{ Mal coasts } & Milicia excelsa & 1 & 3 & 0,3 & 3 & 224 & 0,01 & 0,32 & 1 \\
\hline & Myrianthus arboreus & 1 & 3 & 0,3 & 3 & 224 & 0,01 & 0,32 & 1 \\
\hline \multirow{4}{*}{ Gastritis } & Myrianthus arboreus & 1 & 3 & 0,3 & 1 & 224 & 0 & 0,33 & 1 \\
\hline & Hunteria umbellata & 1 & 3 & 0,3 & 4 & 224 & 0,02 & 0,32 & 1 \\
\hline & Terminalia superba & 1 & 3 & 0,3 & 4 & 224 & 0,02 & 0,32 & 1 \\
\hline & Baillonella toxisperma & 1 & 7 & 0,1 & 1 & 224 & 0 & 0,14 & 1 \\
\hline $\begin{array}{ll}\text { Against seasonal } \\
\text { diseases }\end{array}$ & Triplochiton scleroxylon & 1 & 3 & 0,3 & 1 & 224 & 0 & 0,33 & 1 \\
\hline \multirow[t]{2}{*}{ Headache } & Cola sp. & 1 & 3 & 0,3 & 4 & 224 & 0,02 & 0,32 & 1 \\
\hline & Scorodophloeus zenkeri & 1 & 4 & 0,3 & 4 & 224 & 0,02 & 0,23 & 1 \\
\hline \multirow[t]{4}{*}{ Stomach aches } & Ficus muсuso & 1 & 3 & 0,3 & 4 & 224 & 0,02 & 0,32 & 1 \\
\hline & Holoptelea grandis & 1 & 1 & 1 & 7 & 224 & 0,03 & 0,97 & 3 \\
\hline & Vepris louisii & 1 & 1 & 1 & 7 & 224 & 0,03 & 0,97 & 3 \\
\hline & Diopyros abissinica & 1 & 2 & 0,5 & 7 & 224 & 0,03 & 0,47 & 2 \\
\hline
\end{tabular}




\begin{tabular}{|c|c|c|c|c|c|c|c|c|c|}
\hline Diseases & Plants & C1 & $\mathrm{C2}$ & P1 & C3 & C4 & $\mathbf{P 2}$ & P1-P2 & Ip \\
\hline \multirow[t]{4}{*}{ Malaria } & Picralima nitida & 1 & 2 & 0,5 & 7 & 224 & 0,03 & 0,47 & 2 \\
\hline & Alstonia boonei & 1 & 6 & 0,2 & 7 & 224 & 0,03 & 0,14 & 1 \\
\hline & Cola sp. & 1 & 3 & 0,3 & 7 & 224 & 0,03 & 0,30 & 1 \\
\hline & Enantia chlorantha & 1 & 3 & 0,3 & 7 & 224 & 0,03 & 0,30 & 1 \\
\hline Lice & Baillonella toxisperma & 2 & 7 & 0,3 & 1 & 224 & 0 & 0,28 & 1 \\
\hline Prevention & Dicranolepis sp. & 1 & 3 & 0,3 & 1 & 224 & 0 & 0,33 & 1 \\
\hline Painful periods & Diospyros crassiflora & 1 & 4 & 0,3 & 2 & 224 & 0,01 & 0,24 & 1 \\
\hline $\begin{array}{l}\text { Regulation of } \\
\text { heartbeat }\end{array}$ & Panda oleosa & 1 & 3 & 0,3 & 1 & 224 & 0 & 0,33 & 1 \\
\hline $\begin{array}{l}\text { Strengthening } \\
\text { sexual virility }\end{array}$ & Swartzia sp. & 1 & 4 & 0,3 & 6 & 224 & 0,03 & 0,22 & 1 \\
\hline Rheumatism & Barteria fistulosa & 1 & 3 & 0,3 & 3 & 224 & 0,01 & 0,32 & 1 \\
\hline Measles & Enantia chlorantha & 1 & 3 & 0,3 & 2 & 224 & 0,01 & 0,32 & 1 \\
\hline \multirow{2}{*}{$\begin{array}{l}\text { Stimulation } \\
\text { mammal milk }\end{array}$} & Ricinodendron heudelotii & 1 & 4 & 0,3 & 4 & 224 & 0,02 & 0,23 & 1 \\
\hline & Anonidium mannii & 1 & 4 & 0,3 & 4 & 224 & 0,02 & 0,23 & 1 \\
\hline \multirow[t]{2}{*}{ Syphilis } & Anonidium mannii & 1 & 4 & 0,3 & 2 & 224 & 0,01 & 0,24 & 1 \\
\hline & Raphia regalis & 1 & 3 & 0,3 & 2 & 224 & 0,01 & 0,32 & 1 \\
\hline Tetanus & Dicranolepis sp. & 1 & 3 & 0,3 & 1 & 224 & 0 & 0,33 & 1 \\
\hline \multirow{3}{*}{ Cough } & Ficus muсиso & 1 & 3 & 0,3 & 14 & 224 & 0,06 & 0,27 & 1 \\
\hline & Klainedoxa gabonensis & 1 & 4 & 0,3 & 14 & 224 & 0,06 & 0,19 & 1 \\
\hline & Ricinodendron heudelotii & 1 & 4 & 0,3 & 14 & 224 & 0,06 & 0,19 & 1 \\
\hline Tuberculosis & Alstonia boonei & 1 & 6 & 0,2 & 1 & 224 & 0 & 0,16 & 1 \\
\hline \multirow[t]{3}{*}{ Intestinal worms } & Alstonia boonei & 1 & 6 & 0,2 & 5 & 224 & 0,02 & 0,14 & 1 \\
\hline & Enantia chlorantha & 1 & 3 & 0,3 & 5 & 224 & 0,02 & 0,31 & 1 \\
\hline & Scorodophloeus zenkeri & 1 & 4 & 0,3 & 5 & 224 & 0,02 & 0,23 & 1 \\
\hline
\end{tabular}

Some plants have an average and high $I p$ rating performance $(I p=1 \& I p=2)$ for some illnesses.

\section{DISCUSSION}

Gribé village is located in a forest area and a total number of 224 recipes based on plants were registered for 101 diseases or symptoms mentioned by the local populations. Almost all the populations of that locality resort to plants for primary healthcare, because of the lack of modern health facilities. Even when women are giving birth, plants are used in order to ease the expulsion of the fetus. The most difficult cases are transferred to the nearest hospital which is situated at Massea (About $20 \mathrm{~km}$ from the village and $56 \mathrm{~km}$ from Yokadouma).

In fact, people who resort to traditional medicine (auto medication and traditional healers) represent more than $98 \%$ of the interviewees. Quantitative data obtained from investigations within households in the locality of Gribé show the interest of the populations in traditional medicine based on plants for primary healthcare. It corroborates with the predictions of the WHO which estimated that $80 \%$ of the rural populations resort to traditional medicine for their primary healthcare (OMS,1983; Adjanohoun et al., 1996; Eddouks et al., 2007). This number is increasing (WHO, 2013; 2014; Orch et al., 2015). The Baka pygmies are those who mentioned the highest number of plants (59.77\%) and recipes as against $37 \%$ to $36 \%$ for the Kounabembe. This illustrates the fact that Baka pygmies are widely known as experts of the forest and pharmacopoeia (Betti \& Lejoly, 2010). The highest contribution by the men $68.10 \%$ as against $31.9 \%$ for the women shows that the men are the most important actors in the knowledge of forest resources.

As concerns the diversity of plants used in the traditional pharmacopoeia the number of treatments of 348 therapeutic indications are considerable with regard to the results obtained in some countries (Agelet \& Vallès, 2001; Mulet, 1990). The great number of plant species used within the study area provides evidence that the local population mainly depend on medicinal plants to get rid of many illnesses. This is a good indicator of the profound knowledge of plants by these populations. The 132 registered species represent $16.09 \%$ of the diversity of the medicinal flora of Cameroon which counts 820 medicinal plants (Nkongmeneck, 2007) and 0.52\% of the Africanflora that is made up of 25000 species (Ouhaddou et al.,2014).

These results are very representative compared to those of countries where there are accompaniment means and considerable time for the collection of data (Villar et al., 1992).

In Cameroon, in addition to the national inventory of medicinal plants made by Adjanohoun et al. (1996), and which enabled that registration of more than 400 species of plants, Dibong et al. (2001) made an inventory of 90 sheds where medicinal plants were being sold in three local markets and registered 30 species. In Benin, Adoumou et al. (2012) recorded 205 species whereas in Morocco, Ouhaddou and his collaborators in 2014 recorded 110 vegetal species in the region of Agadir Ida Ou Tanane. In certain localities, the geo-climatic situation 
contributes to the low availability of medicines derived from plants. That is why the populations are bound to use the plants they have in many forms.

Many groups of persons have varied knowledge on traditional medicine based on plants. That is why this knowledge is widespread within the populations of the locality and this constitutes an indicator that can enable researchers to assess the data of an ethno-botanic study (Johns et al., 1990; Agelet \& Vallès, 2001).

Sexual impotence is an illness that originates mainly from the lack of sexual hygiene. That could be the case in the study area where hygienic conditions are not really respected. It is relatively associated to illnesses, given the fact that is has to do with the enhancement of virility and the upholding of the man's erection during sexual intercourse.

As concerns backaches and related disorders such as lumbagos which are very frequent in forest regions (Betti \& Lejoly, 2010), plants proposed for the treatment are represented in primary healthcare in this area Besides, Baillonella toxisperma, Gambeya lacourtiana, Mansonia altissima, Scorodophloeus zenkeri, Tetrapleura tetraptera (Betti \& Lejoly, 2010). We can also mention Copaifera mildbraedii, Cylicodiscus gabunensis, Diospyros crassiflora, Lecaniodiscus sp. and Leptonychia sp., as species that could be used in the treatment of this illness.

In Africa, diseases such as diarrhea, malaria and pneumonia are the main causes of infant mortality (Black et al., 2010). Sub-Saharan Africa pays the highest price to malaria related mortality given that it accounts for $90 \%$ of deaths (Anonym, 2015). Certain plants are used for many purposes and are involved in the treatment of many diseases in study the area. It is the case with Baillonella toxisperma that treat infertility, backache, gastric disorder, fever and lice Alstonia boonei (gonorrhea, infertility, syphilis, stimulation of milk secretion) Klainedoxa ganonensis (internal wounds, gonorrhea, cough, AIDS) and many other plants. It is also the case of Baillonella toxisperma which is the plant that is mostly mentioned in this locality but also in other regions of Cameroon and Africa (Betti, 2002b; Dijk, 1999; Adjanohoun et al., 1996; Bouquet, 169). Alstonia boonei is also mentioned in many African countries having anti-malaria and anti-fever properties (Betti et al.,2014). Plants belonging to the same species could synthesize the same pharmacological components. For that reason, they could be used in the treatment of the same diseases from one geographical area to the other (Adjanohoun et al., 1996).

Decoction is the method of extraction mostly used within the locality. The majority of active principles need the heat for their extraction whereas the other methods of extraction are mainly mostly destroyed by heat or volatile (Tajeukem, 2004). The barks are the parts that are mostly solicited in plants because their secondary metabolites are mostly stocked in the barks than in the leaves (Ouhaddou et al., 2014). The height of the trees do not enable people to get easy access to the leaves as compared to shrubs, herbs or creeps.

The medicines are mostly absorbed through the oral track $(59.25 \%)$. This track is the most privileged because most of these drugs are in the form of decoctions (Saadi et al., 2013).

The anal track is another gateway to the body through which the administration of medicines leads to the cleansing of the different passage of foods and certain wastes.

The results obtained in the village of Gribé show that the bark and the stem $(73.21 \%)$, the leaves $(8.89 \%)$, the grains $(3.57 \%)$, the sap $(2.68 \%)$ are the parts that are mostly used in the plants. The predominance of barks is the clear indication of the availability of the big trees which are less influenced by the seasons hence, the best means of their conservation (Adomou et al., 2012).

The high rate of the use of leaves could be explained by the fact that these parts are accessible. Moreover, their exposure to the sun favours the photosynthetic activity which favors biosynthesis and the storage of metabolites by the plants (El Rhaffari \& Zaid, 2000). Furthermore, through photosynthesis, the leaves gather other alkaloids which could account for their medicinal proprieties and this explains why they are widely used (Simbo, 2010).

Certain plants such as Enantia chlorantha $(I p=1)$ and Picralima nitida $(I p=2)$ were harvested on the site and their anti-malaria activities are well known after being tested. Betti (2004) made mention of the anti-malaria activity of Enantia chlorantha which was confirmed within the framework of the fight against Plasmodium yoellinigeriensis (Agomo et al., 1992). It goes the same with Picralima nitida in the fight against malaria. Certain plants with a low importance index have anti-microbial activities contrary to others that have a high importance index but in which microbial activities are unknown.

\section{Conclusion}

Studies carried out in the northern periphery of the Boumba-Bek National Park enabled us to realize that any timber forest products can be used as medicinal plants and could be highly involved in primary healthcare through traditional medicine. Plants used as drugs in this locality treat many affections of the various systems of the human body. The drugs used are mostly in their raw state and the extraction is aqueous for decoction, maceration and infusion. Administration is oral, skin application, inhalation or anal. Certain plants such as Baillonella toxisperma, Alstonia boonei, Anonidium mannii, Klainedoxa gabonensis, Ricinodendron heudelotii and Scorodophloeus zenkeri are used to cure many diseases. Other plants are combined to cure some diseases as it is the case with 
abscesses, infertility .... Some diseases such as fever, filarioses, pile and many others have a sole treatment. It would be advisable to multiply studies in order to have a great variety of treatment proposals for all the diseases of that area that can permit the promotion of medicinal plants and pharmaceutical impulsion that will permit the access to the drugs at low price.

\section{Acknowledgments}

We first of all we thank the Japanese Cooperation (JICA) through the FOSAS project for accepting to fund this research. We also thank the inhabitants of Gribé for their truthful collaboration. We would also like to express our special gratitude to Mr. ADAM Marc, Mr. BIAP Mermoz, Mr. BAYAKA Georges and Mr. MIAMBOUO Georges and all those who accompanied us on the field. Our sincere gratitude also goes to Dr Hirai MASAAKI, Dr. HAYASHI, M. ENDO, Mr. ALIDOU as well as to all those who facilitated this research.

\section{References}

Abouri, M., El Mousadik, A., Msanda, F.; Boubaker, H., Saadi, B. \& Cherifi, K. (2012). An ethnobotanical survey of medicinal plants used in the Tata Province, Morocco. International Journal of Medicinal Plants Research 1(7): 99-123

Adjanohoun, J. E., Aboubakar, N., Dramane, K., Abot, M. E., Ekpere, J. A., Enow-Orock, K. G., Focho, D., Gbile, Z. O., Kamanyi, A., Kamsu Kom, J., Keita, A., Mbenkum, T., Mbi, C. N., Mbiele, A. L., Mbome, I. L., Mubiru, N. K.; Nancy, W. L.; Nkongmeneck, B.; Satabi, B. ; Sofowora, A.; Tamze, V.; \& Wirman, C. K. (1996). Traditional medicine and pharmacopoeia: contribution to ethnobotanical and floristic studies in Cameroon. Organisation of African Unity. Scientific, technical and research commission (OAU/STRC) Lagos. 485P.

Adomou, A. C.; Yedomonhan, H.; Djossa, B.; Legba, S. I.; Oumorou, M. \& Akoegninou, A. (2012). Etude Ethnobotanique des plantes médicinales vendues dans le marchéd'Abomey-Calavi au Bénin Int. J. Biol. Chem. Sci. 6(2): 745-772

Agelet, A. \& Vallès, J. (2001). Studies on pharmaceutical ethnobotany in the region of Pallars (Pyrenees, Catalonia, Iberian Peninsula). Part I. General results and new or very rare medicinal plants. Journal of Ethnopharmacology 77. Pp 57-70

Agomo, P.U., J.C. Idigo \& B.M. Afolabi (1992). Antimalarial medicinal plants and their impact on cell populations in various organs of mice. African Journal of Medicine and Medical Sciences, 21 (2): 39-46.

Anonyme (2015). Projet d'immersion en communauté 2007 : lutte contre le paludisme dans le cadre d'un hôpital périphérique. http://www.medecine.unige.ch

Betti J. L. (2004). An ethnobotanical study of medicinal plants among the Baka pygmies in the Dja Biosphere Reserve, Cameroon. African Study Monographs, 25(1): 1-27

Betti, J. L. \& Lejoly, J. (2010). Contribution à la connaissance des plantes médicinales de la réserve de biosphère du Dja au Cameroun : plantes utilisées dans le traitement des maux de dos. Int. J. Biol. Chem. Sci. 4(1): 193200.

Betti, J. L. (2002b). Medicinal plants sold in Yaoundé markets, Cameroon. African Study Monographs, 23 (2): $47-$ 64.

Betti, J. L., Caspa Gua, R., Ambara, J., Kourougue R. L. \& Eboule A. (2014). Usages de Alstonia boonei de Wild (Apocynaceae) en médecine traditionnelle à Mbeth II (Est-Cameroun) in Progress report 2013. ForestSavannah sustainability project, Cameroon. pp. 215-224

Betti, J. L. (1996). Etude ethnobotanique des plantes médicinales de l'ensemble de la réserve de faune du Dja et sa périphérie : plantes médicinales utilisées par les guérisseurs. Rapport technique Projet ECOFAC (Conservation et utilisation rationnelle des écosystèmes forestiers en Afrique centrale), AGRECO - CTFT, Bruxelles, $17 \mathrm{p}$.

Black, R. E., Cousens. S., Johnson, H.L., Lawn, J. E., Rudan, I., Bassani, D. G., Jha, P., Campbell, H., Walker, C. F., Cibulskis, R., Eisele, T., Liu, L. \& Mathers, C. (2010). Global, regional, and national causes of child mortality in 2008: a systematic analysis. The Lancet, 375 (9730): 1969-1987

Bouquet, A. (1969). Féticheurs et médecines traditionnelles du Congo-Brazzaville. Mémoire RSTOM n 32, Paris.

Diame, G. L. A. (2010). Ethnobotany and ecological studies of plants used for reproductive health: a case study at Bia biosphere reserve in the western region of Ghana. University of Cape Coast Ghana.Finalreport.UNESCO (MAB) Young Scientist Research Award Scheme Paris Cedex 15 France.125p.

Dibong, S. D., MpondoMpondo, E., Ngoye, A., Kwin, M. F. \& Betti, J. L. (2011). Ethnobotanique et phytomédecine des plantes médicinales de Douala, Cameroun. Journal of Applied Biosciences, 37 : 24962507.

Dijk, J. F. W. (1999). Non-timber forest products in the Bipindi-Akom II region, Cameroon.A socio-économic and ecological assessment.TheTropenbos-Cameroon programme.

Eddouks, M., Ouahidi, L., Farid, O., Moufid, A., Khalidi, A. \& Lemhadri, A. (2007). Utilisation des plantes 
médicinales dans le traitement du diabète au Maroc. Phytothérapie 5 : 194-203.

El Rhaffari, U. \& Zaid, A. (2000). Pratique de la phytothérapie dans le sud-est du Maroc (Tafilalet). Un savoir empirique pour une pharmacopée rénovée. Des sources du savoir aux médicaments du futur. IRD Editions. Actes du 4ème Congrès européen d'ethnopharmacologie, 11-13 mai 2000, Metz (France).

FAO, IFAD \& WFP. (2015). The State of Food Insecurity in the World 2015. Meeting the 2015 international hunger targets: taking stock of uneven progress. Rome, FAO.

FAO. (2010). Global forest resources assessment 2010. Progress towards sustainable forest management. Rome, FAO (Available at: www.fao.org/docrep/013/i1757e/i1757e.pdf).

FAO. (2013). The Contribution of Forests to Sustainable Diets. Background paper for the International Conference on Forests for Food Security and Nutrition. Rome, FAO.

Helms, J. A. (1998). Dictionary of forestry.Society of American foresters and CAB publishing, Willing ford, Royaume-Uni. 210P.

Hirai, M. (2014). Agricultural land use, collection and sales of Non-Timber Forest Products in the agroforest zone in southeastern Cameroon. African Study Monographs, Suppl. 49: 169-202

Jamnadass, R. H., Dawson, I. K., Franzel, S., Leakey, R. R. B., Mithhöfer, D., Akinnifesi, F.K. \& Tchoundjeu, Z. (2011). Improving livelihoods and nutrition in sub-Saharan Africa through the promotion of indigenous and exotic fruit production in smallholders' agroforestry systems. International Forestry Review, 13(3): 338-354.

Johns, T., Kokwaro, J.O. \& Kimanani, E. K., (1990). Herbal remedies of the Luo of Siaya District, Kenya: establishing quantitative criteria for consensus. Economic Botany 44 (3), 369-381.

Koyeu, T. E., Mendi, G., Tchamago, F. X., Tajeukem, V. C., Franklin, V. \& Linda, C. (2014). Ethnobotanic Contribution of Cameroon: Anti-Diabetic Plants Inventory in the Nkoung-Khi Division West Region Cameroon. App. Sci. Report. 8 (3), 2014 : 125-133

Lejoly, J. (1993). Ethnobotanique et méthodologie pour les inventaires biologiques. Projet ECOFAC, AGRECOCTFT, Bruxelles, 137 p.

Letouzey, R. (1968). Etude phytogéographique du Cameroun. In (P. Lechevalier, ed.) Encyclopédie Biologique, $L X I X$, p. 511. P. Lechevalier, Paris.

Letouzey, R. (1985). Notice de la Carte Phytogéographique du Cameroun au 1: 500000. Institut de la Carte Internationale de la Végétation, Toulouse.

Mulet, L. 1990. Aportaciones al conocimientoetnobota'nico de la provincia de Castellón. Doctoral Thesis, Faculty of Pharmacy, University of Vale'ncia, $862 \mathrm{p}$.

Nicolas J. P., K. Chikh, N. Lebeurier \& Meunier Q. (2004). Savoirs traditionnels et santé communautaires: la stratégie alternative de « Jardins du Monde ».

Nkongmeneck, B. A. (2007a). Répertoire des plantes médicinales du Cameroun. Cameroon journal of ethnobotany. Vol.1 N² PP. 36-46

Nkongmeneck, B. A. (2007b). Collaboration tradithérapeutes-médecins. Cameroon journal of ethnobotany. Vol.1 $\mathrm{N}^{\circ} 2$ pp. $57-63$

Nkongmeneck, B. A., Jiofack, T. R. \& Kemeuze, V. A. (2007) : Valorisation des ressources biologiques de la réserve de biosphère du Dja (Cameroun) Projet MEM/UNESCO, rapport technique $\mathrm{n}^{\circ} 1.33 \mathrm{P}$.

O.M.S. (1983). Médecine traditionnelle et couverture des soins de santé. O.M.S., Genève, Suisse, 335 p.

OMS. (2012). Classification Statistique Internationale des Maladies et des Problèmes de Santé Connexes. Deuxième version. Institut canadien d'information sur la santé. CIM/10/CA/CCI. 1027p.

OMS. (2013). Stratégie de l'OMS pour la médecine traditionnelle pour 2014-2023. 72p.

OMS. (2014). Une décennie d'action de l'OMS dans la Région africaine Euvrer ensemble pour atteindre les objectifs en matière de santé. Bureau OMS pour l'Afrique. $108 \mathrm{p}$.

Orch, H.; Douira, A. \& Zidane, L. (2015). Étude ethnobotanique des plantes médicinales utilisées dans le traitement du diabète, et des maladies cardiaques dans la région d'Izarène (Nord du Maroc). ISSN 1997-5902. Journal of Applied Biosciences 86:7940-7956

Ouhaddou, H., Boubaker, H., Msanda F. \& El Mousadik A. (2014). An Ethnobotanical Study of Medicinal Plants of the Agadir Ida OuTanane Province (Southwest Morocco). Journal of Applied Biosciences 84:7707-7722.

Saadi, B., Msanda, F. \& Boubaker, H. (2013). Contributions of folk medicine knowledge in south-western Morocco: The case of rural communities of Immouzzer Ida OuTanane Region. International Journal of Medicinal Plant Research pp. 135-145.

Salmerón-Manzano, E., Garrido-Cardenas J. A. \& Manzano-Agugliaro F. (2020). Worldwide Research Trends on Medicinal Plants. International Journal of Environmental Research and Public Health. MDPI 17, 3376. 20p.

Simbo, D. J. (2010). An ethnobotanical survey of medicinal plants in Babungo, Northwest Region, Cameroon. Journal of Ethnobiology and Ethnomedicine, 6:8.

Sunderland, T. C. H. (2011). Food security: why is biodiversity important? International Forestry Review, 13(3): 265-274.

Tajeukem, V. C., Fongnzossie Fedoung, E., Kemeuze V. A. \& Nkongmeneck, B. A. (2014). Vegetation Structure 
and Species Composition at the Northern Periphery of the Boumba-Bek National Park, Southeastern Cameroon. African Study Monographs Supplementary Issue, 49: 13-46.

Tchatat, M., Ndoye, O. (2006). Etude des produits forestiers non ligneux d'Afrique Centrale : réalités et perspectives. Bois et forêts des tropiques. Le point sur les produits forestiers non ligneux. Document de projet $\mathrm{n}^{\circ} 288(2) 2$.

Toda, M. (2014). People and social organizations in Gribe, southeastern Cameroon.African Study Monographs Supplementary Issue, 49: 139-168.

Villar, L., Palacı'n, J.M., Calvo, C., Go'mez, D. \& Montserrat, G. (1992). Plantas medicinales del Pirineoaragone's y dema'stierras oscenses. Diputacio'n de Huesca, Huesca, 2nd ed. 322 p.

Werner, D. (2002). Là où il n'y a pas de Docteur. Enda Tiers-Monde, Dakar. 3ème édition. 597p. 